\title{
Elaboração de material didático para educação em saúde direcionado para hipertensão arterial
}

\author{
Antonio Nildo Bento da Silva1', Eugênio Santana Franco², Edilson Martins Rodrigues Neto³, \\ Márcia Oliveira Coelho ${ }^{4}$, Jani Cleria Pereira Bezerra ${ }^{5}$
}

\begin{abstract}
RESUMO
A educação em saúde (ES) representa um importante instrumento facilitador para a capacitação da comunidade, contribuindo para a promoção da saúde. 0 estudo tem como objetivo, apresentar a elaboração de uma ferramenta de Educação em Saúde direcionada ao combate da Hipertensão Arterial Sistêmica (HAS), assim reforçar a importância de Educação em Saúde no controle das doenças crônicas. Trata-se de uma proposta para construção um material educativo (Círculo da Hipertensão) com orientações aos cuidados com a HAS. Acredita-se ter se criado uma maneira de tornar a ES mais interativa e presente para população mais desinformada ou com dificuldade de aderir à mudança de estilo de vida na prevenção e/ou complicação da HAS. Considera-se que o instrumente cumpre sua finalidade sendo uma ferramenta de fácil construção, de baixo custo e pedagógica, podendo ser relevante no acompanhamento de pacientes hipertensos.
\end{abstract}

Descritores: Educação em Saúde; Hipertensão; Educação de Paciente como Assunto.

\section{Material development for teaching health education directed to hypertension}

\begin{abstract}
Health Education (HE) is an important instrument to facilitate the training of the community, contributing to the promotion of health. To present the development of a health educational tool aimed at combating the systemic arterial hypertension (SAH), thus reinforcing the importance of Health Education in combating chronic diseases. This is a proposal to build an educational stuff (Circle of Hypertension) providing guidelines for care SAH. It is believed to have created a way to make this more interactive and for more uninformed or hard to adhere to the change of lifestyle in the prevention and / or complication of SAH population HE. It is considered that the instrumente fulfills its purpose being a tool of easy construction, low cost and pedagogical, and may be relevant in the monitoring of hypertensive patients.
\end{abstract}

Descriptors: Health Education; Hypertension; Patient Education as Topic.

${ }^{1}$ Graduado em Enfermagem pela Faculdade Integrada da Grande Fortaleza (FGF), Fortaleza, CE, Brasil.

${ }^{2}$ Doutor em Enfermagem Clínico - Cirúrgica pela Universidade Federal do Ceará (UFC), Fortaleza, CE, Brasil.

${ }^{3}$ Mestre em farmacologia pela Universidade Federal do Ceará (UFC), Fortaleza, CE, Brasil.

${ }^{4}$ Mestre em Saúde Pública pela Universidade Estadual do Ceará (UECE), Fortaleza, CE, Brasil.

${ }^{5}$ Doutora Ph. D. em Medicina do Esporte pela Universidad Católica Nuestra Señora de la Asunción (UC), Asunción, Paraguai. 


\section{Introdução}

A hipertensão arterial sistêmica (HAS) é uma doença crônica complexa, multicausal e multifatorial que se caracteriza como um problema de saúde pública em todo o mundo. No Brasil estima-se que 15 a $20 \%$ da população adulta apresenta esta doença, sendo que, na população idosa a prevalência é de $65 \%$. Esta é uma patologia que representa um elevado custo social, pois é responsável por cerca de $40 \%$ dos casos de aposentadoria precoce e de faltas no trabalho ${ }^{1}$.

As doenças crônicas figuram como principal causa de mortalidade e incapacidade no mundo. Elas são mais predominantes em países desenvolvidos, sendo fatores causadores relevantes: o estilo de vida, tabagismo, etilismo, dieta e sedentarismo. Estima-se que cerca de 59\% dos 56,5 milhões de óbitos anuais ocorrem devido aos chamados agravos não transmissíveis que incluem, diabetes, obesidade, doenças respiratórias, câncer e doenças cardiovasculares, como a Hipertensão Arterial Sistêmica ${ }^{2,3}$.

A Hipertensão Arterial Sistêmica (HAS) é a mais freqüente das doenças cardiovasculares. É também o principal fator de risco para as complicações mais comuns como acidente vascular cerebral e infarto agudo do miocárdio, além da doença renal crônica terminal ${ }^{4}$.

A HAS apresenta alta prevalência na população brasileira, influenciando na gênese de doenças cerebrovasculares, coronarianas, doença de retina, insuficiência cardíaca, insuficiência renal crônica e doenças vasculares. O reconhecimento do aumento da prevalência da HAS na população jovem e de suas possíveis complicações na vida adulta tem implicações importantes para a prevenção de doenças crônicas, em especial as cardiovasculares ${ }^{5}$.

Sendo a HAS uma doença, na maior parte do seu curso, assintomática, tem seu diagnóstico e tratamento, freqüentemente, negligenciado, somando-se a isso a baixa adesão, por parte do paciente, ao tratamento prescrito. Estes são os principais fatores que determinam um controle muito baixo da HAS aos níveis considerados normais em todo o mundo, a despeito dos diversos protocolos e recomendações existentes e um maior acesso a medicamentos ${ }^{4}$.

Vários fatores têm sido correlacionados com as cifras de pressão arterial, sendo as principais: a condição sócio-econômica, a etnia, os hábitos alimentares, a idade, o estado de vigília e sono, a agregação familiar, o peso e a estatura ${ }^{6}$.

Numa comparação com gerações anteriores às mudanças nos hábitos alimentares com consumo excessivo de alimentos ricos em gordura saturada, bebidas hipercalóricas, associados aos baixos níveis de atividade física, determinam uma pandemia de sobrepeso e obesidade, e suas consequentes comorbidades como as doenças cardiovasculares ${ }^{7}$.

O diagnóstico da HAS depende de uma avaliação criteriosa, procurando-se identificar a presença de HAS secundária, assim como os portadores de hipertensão de consultório, condição relativamente freqüente nos extremos da idade. Além disso, o diagnóstico da HAS em populações mais jovens, incluindo crianças e adolescentes, tem se tornado mais freqüente, em virtude das altas taxas de obesidade encontradas ${ }^{8}$.

Estratégias de saúde pública são necessárias para a abordagem desses fatores relativos a hábitos e estilos de vida, e, assim, reduzir o risco de exposição, trazendo benefícios individuais e coletivos para a prevenção da HAS e redução da carga de doenças devida às doenças cardiovasculares em geral ${ }^{4}$.

A educação em saúde (ES) representa um importante instrumento facilitador para a capacitação da comunidade, contribuindo para a promoção da saúde, permitindo que trabalhadores de saúde e usuários estabeleçam uma relação dialógica pautada na escuta terapêutica, no respeito e na valorização das experiências, das histórias de vida e da visão de mundo. Para desenvolver estas ações, é necessário o conhecimento das práticas educativas por parte destes trabalhadores, contribuindo, assim, para a redução de várias doenças, principalmente as crônicas 9 .

Os profissionais de saúde que acompanham pessoas hipertensas e com outros problemas de saúde, possuem papel fundamental no planejamento e implementação de estratégias educativas com a finalidade de conduzi-las ao estilo de vida saudável, eliminando ou controlando os riscos da HAS e de outros problemas de saúde entre os familiares, principalmente aqueles hereditários, além do controle efetivo dessas enfermidades ${ }^{10}$.

Diante de tais considerações da importância das estratégias de prevenção no controle da hipertensão arterial, buscase nesse estudo apresentar uma proposta de ferramenta de educação em saúde voltada para HAS. 


\section{Metodologia}

\section{Etapas do desenvolvimento}

Idéia Inicial

A idéia de criar este instrumento originou-se a partir na disciplina de Estágio Supervisionado I do Curso de Enfermagem da Faculdade Integrada da Grande Fortaleza, realizado em uma Unidade da Atenção Primária à Saúde - UAPS do município de Fortaleza-CE no período de setembro a novembro de 2011.

A ideia inicial surgiu durante as atividades de educação em saúde voltadas para a HAS, que são realizadas como instrumento de prevenção e controle pelos profissionais da Estratégia Saúde da Família-ESF devido a compreensão da elevada incidência de portadores de HAS, como um problema de saúde pública em todo o mundo. Observou-se que existe um esforço por parte dos profissionais da ESF para o repasse de informações de modo que favoreça as reais mudanças necessárias para um estilo de vida mais saudável. Com isso, pensou-se em uma maneira de tornar esse processo mais interativo e pedagógico para população pouco informada ou com dificuldade de aderir à mudança de estilo de vida na prevenção e/ou complicação da HAS.

O instrumento foi inspirado no disco obstétrico/gestograma usado pelos profissionais de saúde que realizam consulta de pré-natal, para auxiliar na estimativa da idade gestacional (BRASIL, 2012). O gestograma é de fácil utilização proporcionando rapidez no atendimento ao pré-natal, no qual a partir das informações sobre o primeiro dia da última menstruação é preciso girar a roda da gravidez, para se obter as informações da semana gestacional.

Baseado na tecnologia do disco obstétrico/gestograma, imaginou-se um instrumento que partindo do diagnóstico de classificação da doença hipertensiva, as condutas necessárias para o controle e prevenção. Tais condutas seriam voltadas para as necessidades individuais, bem como, para orientações coletivas.

Com referência dos níveis pressóricos e diagnósticos, utilizou-se o parâmetro de classificação do Ministério da Saúde: Brasil. Ministério da Saúde. Secretaria de Atenção à Saúde. Departamento de Atenção Básica. Hipertensão arterial sistêmica para o Sistema Único de Saúde / Ministério da Saúde, Secretaria de Atenção à Saúde, Departamento de Atenção Básica. - Brasília : Ministério da Saúde, $2006^{11}$.

\section{Materiais e Métodos}

O layout foi criado usando o programa Corel Draw! Versão X5, da Empresa Corel囚. Tendo dimensão de um CD (Compact Disc) convencional. Após a arte pronta, foi impressa, recortada, plastificada e montada as duas partes sobrepostas. A fixação do disco menor sobre o maior ocorreu, inicialmente, com um pedaço de arame recoberto de plástico, após o mesmo foi fixado com ilhóis.

\section{$\underline{\text { Utilização }}$}

A ferramenta está em processo de registro junto à Biblioteca Nacional, com o protocolo de entrada de pedido de patente: BR $202014003965-7$ de 20 de fevereiro de 2014 no Instituto Nacional de Proteção Industrial - INPI, no qual favorecerá a sua utilização nas áreas da saúde e educação nas escolas de nível fundamental e médio como instrumento de Educação em Saúde na prevenção ou acompanhamento da HAS.

\section{Resultados}

A confecção do material educativo passou pelas seguintes etapas. Para facilitar a sua caracterização foi chamado de Círculo Educativo da HAS - CEHAS:

19 Etapa: A Estrutura Física

O CEHAS é composto de 3 partes descritas a seguir: 


\section{- Parte 01.}

$\checkmark$ Dimensões: 0 primeiro disco possui $06 \mathrm{~cm}$ de raio.

$\checkmark$ Material: Esse disco que pode ser feito de vários tipos de materiais tais como: cartolina, papel, plástico e polietileno. Ao ser impresso em material pouco resistente, pode ser plastificado para a proteção do impresso.

- Ilustrações: No circulo maior, em sua metade superior, está impresso os valores Pressão Sistólica: 80, 90, 100, 110, 120, 130, 140, 150 e 160; e os valores limite da Pressão Diastólica: 80, 90 e 100.

Na metade inferior, estão impressos quadros de alerta e quadros da condição hipertensiva, contendo textos que servirão de orientação de conduta para o consultor do círculo. llustrados e exposto no sentido anti-horário, da seguinte forma:

$\checkmark$ Quadro de alerta 01: Com fundo vermelho e letras brancas, contendo o texto: "Procurar Assistência Hospitalar com Urgência!".

$\checkmark$ Quadro de alerta 02: Com fundo laranja e letras pretas, contendo o texto: "Consultar médico!".

$\checkmark$ Quadro de alerta 03: Com fundo róseo e letras pretas, contendo o texto: "Mudar Estilo de Vida!".

$\checkmark$ Quadro de alerta 04: Com fundo verde e letras brancas, contendo o texto: "Parabéns!".

$\checkmark$ Quadro de alerta 05: Com fundo amarelo e letras pretas, contendo o texto: "Atenção!".

$\checkmark$ Quadro de alerta 06: Com fundo azul e letras pretas, contendo o texto: "Procurar Assistência Hospitalar!".

$\checkmark$ Quadro da Condição Hipertensiva 01: Este quadro é dividido em três caixas de textos com as seguintes características:

- Quadro 01: Com fundo branco e letras pretas, contendo o texto: "Hipertensão!".

- Quadro 02: Com fundo laranja e letras pretas, contendo o texto: "estágio 01!".

- Quadro 03: Com fundo vermelho e letras brancas, contendo o texto: "estágio 02!".

$\checkmark$ Quadro da Condição Hipertensiva 02: Com fundo róseo e letras pretas, contendo o texto: "Pré hipertenso!".

$\checkmark$ Quadro da Condição Hipertensiva 03: Com fundo verde e letras pretas, contendo o texto: "IDEAL".

$\checkmark$ Quadro da Condição Hipertensiva 04: Com fundo amarelo e letras pretas, contendo o texto: "Pressão Baixa!".

\section{- Parte 02.}

$\checkmark$ Dimensões: 0 segundo disco possui $4,25 \mathrm{~cm}$ de raio.

$\checkmark$ Material: O material que pode ser confeccionado esse segundo disco (disco menor) são os mesmo do disco maior.

- llustrações: As ilustrações contidas na parte 02 (disco menor) são destinadas às orientações acerca da mudança de estilo de vida a ser seguida pelo consultor do disco. A primeira ilustração exposta de cima para baixo constitui-se de um desenho de um homem praticando exercícios físicos, seguindo do texto: "Praticar exercícios físicos". Já segunda ilustração constitui-se de um menino comendo frutas e verduras, seguindo do texto: "Comer frutas e verduras". A terceira ilustração constitui-se de um homem comendo um espetinho, seguido do texto: "Evitar frituras e gorduras". A quarta ilustração, constitui-se de um saleiro, seguido do texto "Evitar o sal". A quinta ilustração, constitui-se de uma xícara de café, seguido do texto "Evitar café (moderado)". A sexta ilustração constitui-se de um cigarro sobreposto de um círculo vermelho com uma faixa transversal, seguida do seguinte texto: "Evitar o cigarro".

$\checkmark$ Setas guia: A partir do centro da parte 03 estão ilustrada três setas: a seta 01 (azul), a seta 02 (vermelha), e a seta 03 (amarela). As setas 01 e 02 apontam em direções opostas e são perpendiculares à seta 03.

\section{- Parte 03.}

Essa parte é composta de um fixador (ilhóis) que permite a fixação do disco menor sobre o maior, assim como, a possibilidade da movimentação da segunda parte (disco menor) sobre o disco maior, em ambos os sentidos, horário e anti-horário.

$\checkmark$ Dimensões: $O$ ilhóis possui $0,08 \mathrm{~cm}$ de diâmetro.

$\checkmark$ Material: O ilhóis pode ser de alumínio ou latão.

\section{$\underline{2^{a} \text { Etapa: O Funcionamento. }}$}

O funcionamento do CEHAS ocorre da seguinte forma: 
O círculo menor terá que estar com a seta 01 (azul) apontada para o valor 120/80 (120 Sistólica e 80 diastólica). A partir desta posição a leitura deverá ser realizada.

Na sua porção superior, o círculo maior traz os valores padrões da PA, tanto da Sistólica (máxima) quanto da Diastólica (mínima). Deslizando a seta 01, tanto no sentido horário como anti-horário, se tem a condição do indivíduo ao qual está consultando o Círculo, mostrada pela seta 02 , na parte inferior do círculo maior.

O resultado apresentado através da seta 02 na parte inferior do círculo maior é a condição hipertensiva do consultor (pessoa que está consultando a ferramenta). Ela está baseada nos valores dados pelo Ministério da Saúde (MS).

\section{Posições e resultados}

Com relação às posições dispostas na ferramenta, podem-se ter as seguintes posições com seus respectivos resultados:

Na posição inicial recomendado para a leitura: a seta 02 está apontando na parte inferior do círculo maior, para a palavra "IDEAL", por ser esta a PA considerada ideal pelo MS; e ao mesmo tempo a seta 03 estará apontando para a palavra "PARABÉNS", como forma de incentivar o usuário á manter esse padrão.

Na figura 01 segue a ilustração da posição 02 , a partir da posição 01 em um deslocamento da seta 01 no sentido horário, onde se verifica a seta apontada entre os valores 120/80 e 140/90. Nessa posição, a seta 02 aponta para 0 quadro com o texto: "Pré-Hipertenso". Ao mesmo tempo observa-se que a seta 03 aponta para "Mudar estilo de Vida!".

Figura 01 - Posição 02: Situação Pré Hipertensiva

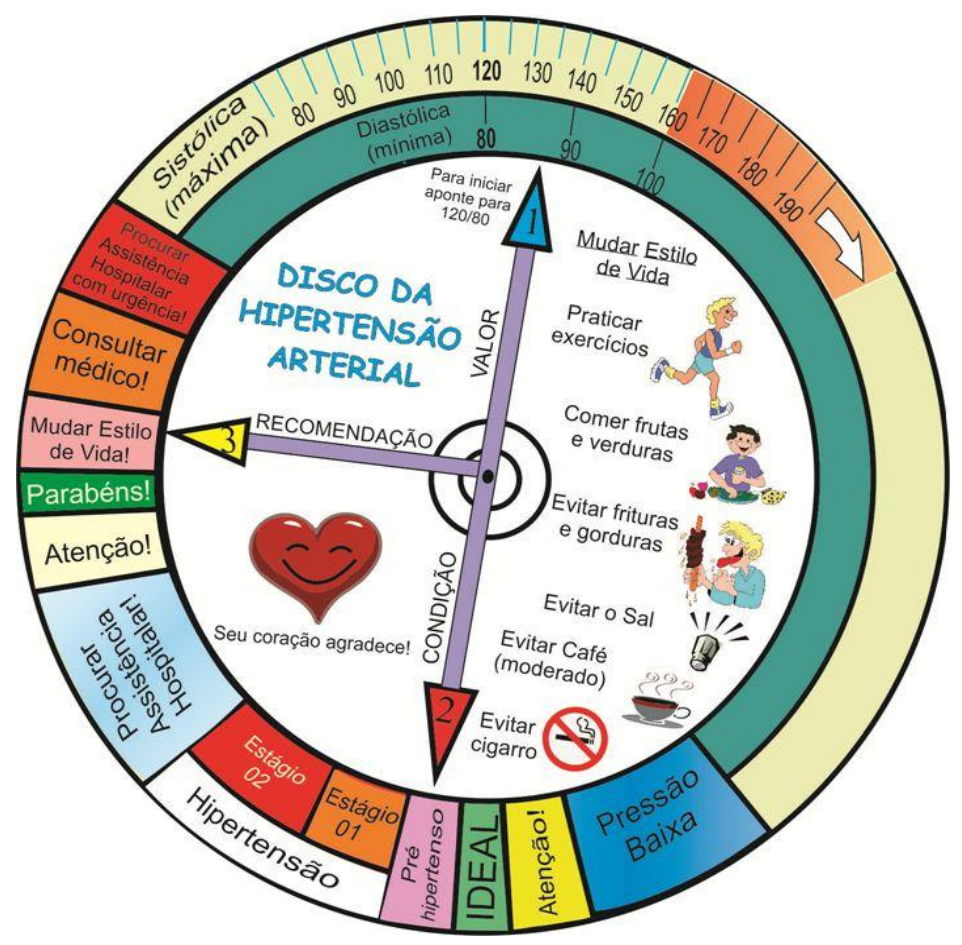

Na figura 02 segue a ilustração da posição 03, a partir da posição 02 em um deslocamento da seta 01 no sentido horário, onde se verifica a seta apontada entre os valores 140/90 e 160/100. Nessa posição, a seta 02 aponta para o quadro com 0 texto: "Hipertenso estágio 01". Ao mesmo tempo observa-se que a seta 03 aponta para "Consultar o médico!".

Na figura 03 segue a ilustração da posição 04, a partir da posição 03 em um deslocamento da seta 01 no sentido horário, onde se verifica a seta apontada para qualquer valor acima de 160/100. Nessa posição a seta 02 aponta para o quadro com o texto: "Hipertenso estágio 02". Ao mesmo tempo observa-se que a seta 03 aponta para "Procurar Assistência Hospitalar com urgência!".

$\mathrm{Na}$ figura 04 segue a ilustração da posição 05 , a partir da posição 04 em um deslocamento da seta 01 no sentido anti-horário, onde se verifica a seta apontada para qualquer valor abaixo de 120/80 e acima que 100 da sistólica. Nessa posição a seta 02 aponta para o quadro com o texto: "Atenção!". Ao mesmo tempo observa-se que a seta 03 aponta para "Atenção!". Alertando o usuário a estar alerta a fim de evitar uma hipotensão. 
Na figura 05 segue a ilustração da posição 06, onde da seta 01 desloca-se no sentido anti-horário a partir da posição 05, apontando para qualquer valor abaixo de 100 da Sistólica, representando uma situação de hipotensão. Nesta posição a seta 01 aponta para o texto "Pressão Baixa!". Ao mesmo tempo, a seta 03 aponta para: "Procurar Assistência Hospitalar!"

Figura 02 - Posição 03: Situação Hipertensiva Estágio 01

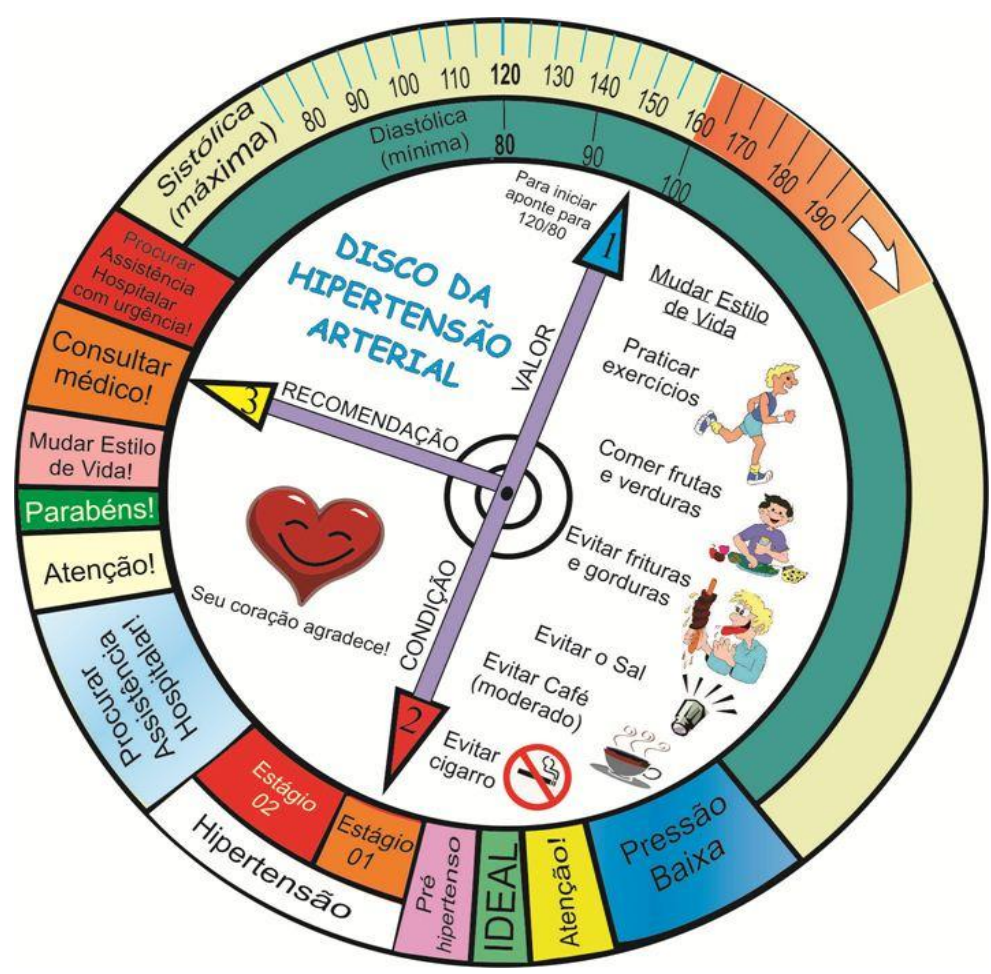

Figura 03 - Posição 04: Situação Hipertensiva Estágio 02

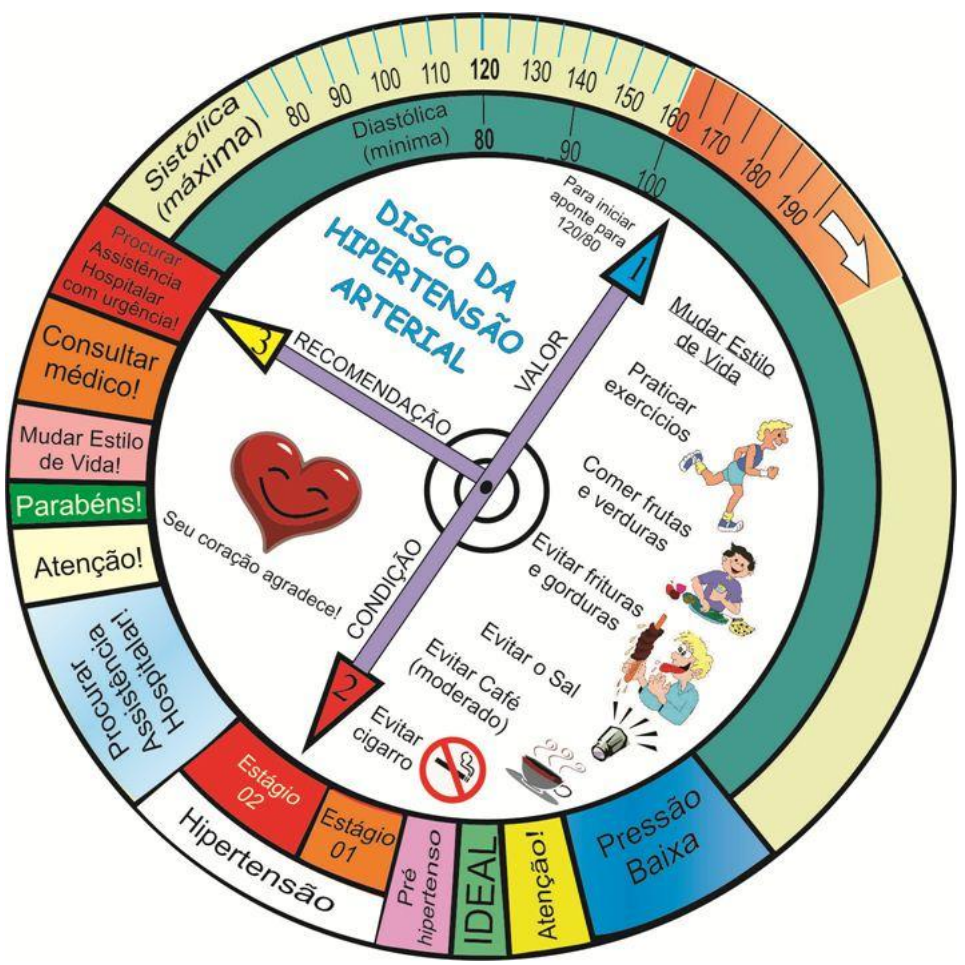


Figura 04 - Posição 05: Situação em Alerta

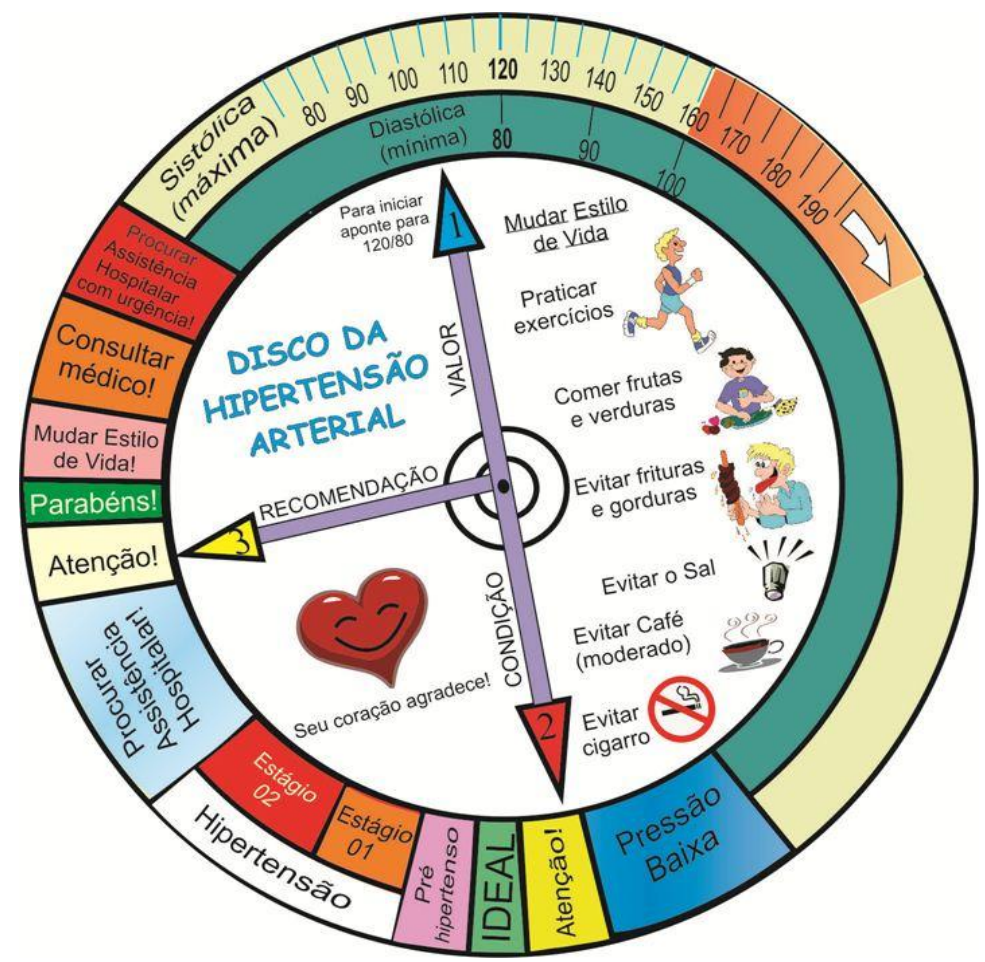

Figura 05 - Posição 06: Hipotensão

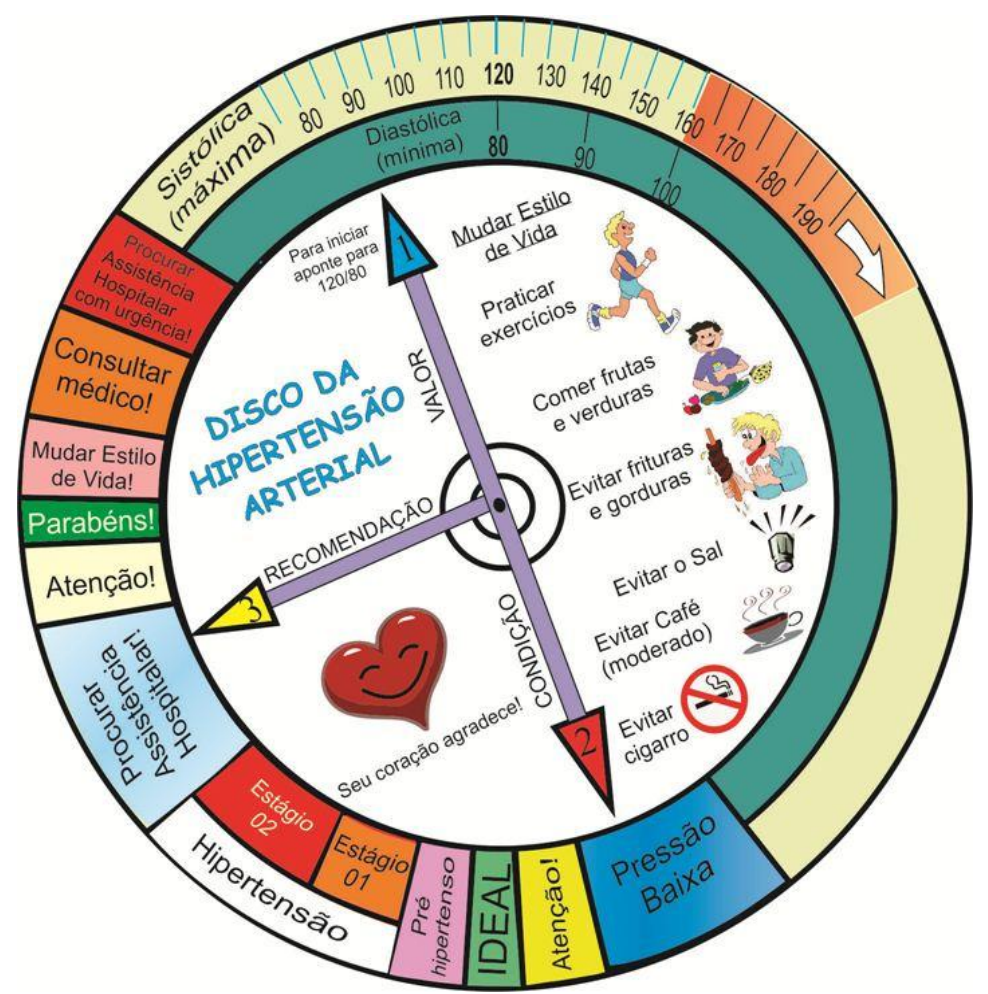

\section{Discussão}

Aplicação prática da Ferramenta nas UAPS.

Esta ferramenta é de extrema utilidade para Educação em Saúde nas UAPS, pois pode ser uma grande aliada tanto no combate como nas complicações da HAS, visto que é um instrumento interativo de fácil utilização e compreensão. 
Autores comentam sobre a importância deste tipo de educação como estratégia para a prevenção da HAS, pois capacita e torna os sujeitos autônomos para tomar as suas decisões, através uma reflexão/ação/reflexão, sobre seu próprio corpo com base nos conhecimentos cada vez mais aprimorados sobre sua saúde, tendo a opção de adotar ou não, hábitos e atitudes saudáveis ${ }^{12}$.

Sobre a utilização de estratégias educativas, ainda citam a necessidade de oficinas ou similares, que possibilitem ao indivíduo compreender a importância da aquisição de conhecimento na seleção e incorporação de atitudes e práticas saudáveis em seu estilo de vida, prevenindo e/ou controlando, desse modo, a síndrome hipertensiva, assim como outros agravos à sua saúde. 12

A seriedade em se pensar em novos tipos de tecnologias e estratégias nas UAPS é reforçada por um estudo em os autores afirmam que a Estratégia de Saúde da Família (ESF) tem como diretriz a promoção da saúde, ficando à equipe de saúde responsável por lançar de todos os esforços para que as mudanças de comportamento para a saúde ocorram no contínuo processo de aprendizagem ${ }^{13}$.

Como estímulo à Criatividade na Educação em Saúde para melhor adesão dos usuários à prevenção ou tratamento, é afirmado que os profissionais de saúde, no âmbito da ESF, necessitam ainda ampliar sua compreensão de educação em saúde e de uso de estratégias educativas que sejam culturalmente significativas para que a participação e decisão de mudanças de comportamento em saúde dos usuários sejam livres e conscientes ${ }^{13}$.

\section{Aplicação prática da Ferramenta nas escolas.}

Essa ferramenta poderá ser usada também nas escolas, onde há frequentemente uma alimentação inadequada e consequentemente riscos das crianças se tornarem adultos hipertensos futuramente.

A ocorrência de HAS foi encontrada em um estudo realizado com 194 crianças com idade entre 6 e 10 anos, em escola em Timóteo, Minas Gerais. Aamostra foi de 22 casos $(11,3 \%)$, destacando a HAS na idade de nove anos, onde a prevalência foi maior entre as idades de 6 a 10 anos, com a freqüência de 8 casos que representam $21,6 \%$ das crianças estudadas nesta faixa etária 7 .

$\mathrm{O}$ resultado da pesquisa mostrou um índice preocupante de HAS em crianças. Esse estudo serviu para que as autoridades em saúde possam desenvolver estratégias voltadas para a prevenção da HAS também em crianças. Nesse contexto CEHAS pode ser usado como ferramenta alternativa para a prevenção da Hipertensão, visto que, por ser bem ilustrativa, interativa e colorida, e de fácil entendimento, possivelmente, será bem aceita pelos estudantes.

Em um estudo sobre a incidência de HAS em uma escola em Pernambuco, foi concluido que o conhecimento da prevalência e dos fatores associados à HAS e à exposição a comportamento de risco por adolescentes poderá estimularlos a exercerem seu papel de modificadores de comportamento na família e na comunidade ${ }^{14}$.

A ideia do autor vem corroborar com essa discussão acerta da importância da Educação em saúde voltada também para o público infanto-juvenil, podendo também ser aliado a educação direcionada à outras doenças crônicas como o Diabetes.

\section{Considerações Finais}

Os usuários do SUS muitas vezes são pessoas com baixa condição sócio-econômica. E isso muitas vezes se reflete também no seu nível educacional. As ações, ferramentas e tecnologias de educação em saúde podem ser um meio com linguagem simples e direta para transmitir conhecimento ao paciente sobre seu estado de saúde.

Acredita-se que a ferramenta apresenta nesse estudo cumpre os requisitos para que possa ser introduzida na rotina da atenção básica como um meio complementar para informação dos pacientes, sobretudo daqueles com menor nível de instrução.

\section{Referências Bibliográficas}

1. SBC; SBH; SBN. Sociedade Brasileira de Cardiologia; Sociedade Brasileira de Hipertensao; Sociedade Brasileira de Nefrologia.VI Diretrizes Brasileiras de Hipertensão. Arq. Bras. Cardiol. v.95, n.1, sup.1, p. 1- 3, 2010.

2. Veras RP. Estratégias para o enfrentamento das doenças crônicas: um modelo em que todos ganham. Rev bras geriatr gerontol, 2011; 14(4): 779-86.

3. Sociedade Brasileira de Hipertensão Arterial, Sociedade Brasileira de Cardiologia, Sociedade Brasileira de Nefrologia. VI Diretrizes Brasileiras de Hipertensão Arterial. Rev Bras Hipertens. 2010;13(1):1-68.

4. Ministério da Saúde. Secretaria de Atenção à Saúde. Departamento de Atenção Básica. Hipertensão arterial sistêmica para o Sistema Único de Saúde / Ministério da Saúde, Secretaria de Atenção à Saúde, Departamento de Atenção Básica. -Brasília : Ministério da Saúde, 2006. 
5. Araújo TL, Lopes MVO, Cavalcante TF, Guedes NG, Moreira RP, Chaves ES, Silva VM. Análise dos indicadores de risco para hipertensão arterial em crianças e adolescentes. Rev Esc Enferm USP. 2008; 42(1):120-6.

6. Pinto, SL, Silva, RDCR, Priore, SE., Assis, AMO, Pinto, EDJ. Prevalência de pré-hipertensão e de hipertensão arterial e avaliação de fatores associados em crianças e adolescentes de escolas públicas de Salvador, Bahia, Brasil. Cad Saúde Pública. 2011; 27(6), 1065-76.

7. Louzada, ANS, Lopes, AGL. Análise da prevalência de hipertensão arterial sistêmica e excesso de peso em crianças da escola municipal do Limoeiro em Timóteo. Revista Funcional. 2009; 1(1), Disponível em: http://www.unilestemg.br/ revistafuncional/arquivos/analise_prevalencia_hipertensao.pdf [acesso 21 jan 2015]

8. ROSA, EC, PLAVNIK, FL, TAVARES, A. Hipertensão arterial sistêmica. Revista Brasileira de Medicina. 2006; 63(1), 5-17. 9. Cervera, DPP; Parreira, BDM; Goulart, BF. Educação em saúde: percepção dos enfermeiros da atenção básica em Uberaba (MG) Ciênc saúde coletiva, 2011; 16(1), 1547-54.

10. SANTOS, ZMSA; LIMA, HP. Ações educativas na prevenção da hipertensão arterial em trabalhadores. Rev. RENE: revista da rede de enfermagem do nordeste. Rede de Enfermagem do Nordeste. V. 9, n. 1, jan./mar., Fortaleza [CE]: A Rede, 2008.

11. BRASIL. Ministério da Saúde. Secretaria de Atenção à Saúde. Departamento de Atenção Básica. Atenção ao prénatal de baixo risco / Ministério da Saúde. Secretaria de Atenção à Saúde. Departamento de Atenção Básica. Brasília: Editora do Ministério da Saúde, 2012.

12. Santos ZMSA, Lima HP. Tecnologia educativa em saúde na prevenção da hipertensão arterial em trabalhadores: análise das mudanças do estilo de vida. Texto \& Contexto Enferm. 2008; 17(1):90- 7. Disponível em: http://www.scielo.br/ pdf/tce/v17n1/10.pdf [acesso 21 jan 2015]

13. Machado, MFAS; Vieira, NFC. Educação em Saúde: o Olhar da Equipe de Saúde da Família e a participação do usuário. Rev. Latino-Am. Enfermagem. 2009; 17(2). Disponível em: http://www.scielo.br/pdf/rlae/v17n2/pt_06.pdf [acesso 21 jan 2015] 14. GOMES, BMR; ALVES, JGB. Prevalência de hipertensão arterial e fatores associados em estudantes de Ensino Médio de escolas públicas da Região Metropolitana do Recife, Pernambuco, Brasil, 2006. Cad. Saúde Pública, 2009; 25(2):375-381.

\section{Antonio Nildo Bento da Silva}

Endereço para correspondência - Rua Capitão Francisco Pedro, n 1210, Bairro: Rodolfo Teofilo, CEP 60430370, Cidade: Fortaleza, CE , Brasil

E-mail: nildob@yahoo.com.br

Lattes: http://lattes.cnpq.br/0972014293955327

Eugênio Santana Franco - esfranco@gmail.com

Edilson Martins Rodrigues Neto - edilsonmrneto@hotmail.com

Márcia Oliveira Coelho - oc.marcia@gmail.com

Jani Cleria Pereira Bezerra - j.cleria@gmail.com

Enviado em 22 de julho de 2014. Aceito em 26 de abril de 2015. 
